\title{
Active-to-sterile neutrino dipole portal and the XENON1T excess
}

\author{
Ian M. Shoemaker $\oplus^{1, *}$ Yu-Dai Tsai $\oplus^{2,3,4, \dagger}$ and Jason Wyenberg $\oplus^{5, \$}$ \\ ${ }^{1}$ Center for Neutrino Physics, Department of Physics, Virginia Tech University, \\ Blacksburg, Virginia 24601, USA \\ ${ }^{2}$ Theory Department, Fermi National Accelerator Laboratory, P. O. Box 500, Batavia, Illinois 60510, USA \\ ${ }^{3}$ Cosmic Physics Center, Fermi National Accelerator Laboratory, Batavia, Illinois 60510, USA \\ ${ }^{4}$ Kavli Institute for Cosmological Physics, University of Chicago, Chicago, Illinois 60637, USA \\ ${ }^{5}$ Department of Physics, University of South Dakota, Vermillion, South Dakota 57069, USA
}

(Received 15 July 2020; accepted 3 December 2021; published 27 December 2021)

\begin{abstract}
In this paper, we find that a magnetic transition dipole moment between tau and sterile neutrinos can account for the XENON1T excess events. Unlike the ordinary neutrino dipole moment, the introduction of the new sterile mass scale allows for astrophysical bounds to be suppressed. Interestingly, the best-fit regions that are compatible with the SN1987A imply either boron-8 as the source flux. We find that sterile neutrinos in the $\sim(500-800) \mathrm{keV}$ mass range are capable of evading astrophysical constraints while being able to successfully explain the XENON1T event rate. We also set new constraints on the dipole portal based on the Xenon 1T data. The sterile neutrino in the best fit parameter space may have significant effects on big bang nucleosynthesis $(\mathrm{BBN})$. We show the region in which a low reheating temperature of the Universe may allow the BBN constraints to be alleviated.
\end{abstract}

DOI: 10.1103/PhysRevD.104.115026

\section{INTRODUCTION}

The nature of particle physics beyond the Standard Model (SM) remains unknown. However, we have two key hints about the nature of new physics: it must account for the nonluminous dark matter (DM), and it must account for neutrino masses. Interestingly, DM direct-detection experiments are sufficiently sensitive to be leading players in searching for novel neutrino interactions that may potentially help solve the mystery of neutrino masses.

This context makes the recent excess of electron recoil events at XENON1T [1] all the more intriguing. Neutrino magnetic moments were originally studied by the XENON1T collaboration as potential explanations for the excess. However, the couplings found tend to exceed the bounds of various astrophysical systems. In this paper, we highlight a neutrino dipole-portal interaction that can account for the signal, while evading astrophysical bounds (though still could be subject to cosmological bounds). This results from the introduction of a new mass scale to the neutrino interaction.

\footnotetext{
shoemaker@vt.edu

†ytsai@fnal.gov

*jason.wyenberg@coyotes.usd.edu
}

Published by the American Physical Society under the terms of the Creative Commons Attribution 4.0 International license. Further distribution of this work must maintain attribution to the author(s) and the published article's title, journal citation, and DOI. Funded by SCOAP.
The most commonly studied models accounting for neutrino masses introduce right-handed sterile neutrinos, $N$, via the interaction $\mathcal{L} \supset H N L$. However, it is important to stress that such singlet states need not dominantly interact with the SM through this particular operator. Viable scenarios exist in which the dominant interaction comes from an active-to-sterile dipole moment, sometimes referred to as the "neutrino dipole portal,"

$$
\mathcal{L} \supset d\left(\bar{\nu}_{L} \sigma_{\mu \nu} F^{\mu \nu} N\right)+\text { H.c., }
$$

where $F_{\mu \nu}$ is the electromagnetic field strength, $\sigma_{\rho \sigma}=$ $\frac{i}{2}\left[\gamma_{\rho}, \gamma_{\sigma}\right], \nu_{L}$ is the SM neutrino, and the coefficient $d$ with units of (mass) $)^{-1}$ controls the strength of the interaction. This transition dipole moment has been studied in the context of MiniBooNE [2-9], and future projected bounds have been studied for IceCube [10], SHiP [8], and directdetection experiments [11]. We note that the SM value of the neutrino magnetic moment was calculated in Refs. [12,13], and is far below the magnitude that XENON1T can probe.

Note that this operator can be induced through loop processes with the ordinary HNL operator, and in most cases, mixing between $N$ and SM neutrinos would also be induced. We focus our attention on the operator in Eq. (1) as a simplified consideration. We also introduce a Dirac mass for $N$, denoted $m_{4}$, and avoid the Majorana mass-term for complications discussed in [8]. In addition, a small mixing angle would not affect our result, and the 
constraints are dependant on the size of the mixing. The tau mixing is not strongly constrained for a $\sim 100 \mathrm{keV}$ sterile neutrino [14-18]. We leave a more complete consideration including the mixing and the potential complications of introducing a Majorana mass term for future work.

We will show that up-scattering from $\nu$ to a heavy sterile neutrino $N$ (with a Dirac mass $m_{4}$ ) within the XENON1T detector can plausibly explain the excess while simultaneously evading astrophysical and other terrestrial bounds. We find that ${ }^{8} \mathrm{~B}$ solar neutrino fluxes lead to a best-fit region, and we can also set new constraints based on the Xenon 1T data. We note that the first direct experimental evidence for neutrinos from the $\mathrm{CNO}$ cycle was just reported by Borexino [19]. Axions, dark photons, other neutrino, and DM proposals to explain the XENON1T excess were also discussed in [20-78]. Reference [79] posits a new light vector-mediator which couples to the SM neutrinos via mass-mixing with a heavy sterile neutrino. Our model is similar, although the present model makes use of few $100 \mathrm{keV}$ sterile neutrinos. Moreover, Ref. [79] has 2 couplings and two masses, whereas the present work contains only one mass and one coupling. As an aside, we note that a possible SM explanation of the XENON1T excess is a Tritium contamination in the experiment. As this is sufficiently discussed in [1], we neglect to comment further on it here.

The remainder of this paper is organized as follows. In Sec. II, we introduce the main framework for computing event rates at XENON1T. In Sec. III we discuss the results of our fit to the XENON1T data under a neutrino dipole interpretation. In Sec. IV we review existing constraints on the model focusing on direct detection, stellar energy loss, supernovae, and BBN. Finally we conclude the paper in Sec. V and discuss future probes of the model.

\section{ENERGY DEPOSITION VIA NEUTRINO DIPOLE PORTAL}

As studied in [11], incoming solar neutrinos can upscatter to the heavy sterile state $N$ in the detector volume of a direct-detection experiment. They will inevitably decay as well through $N \rightarrow \nu+\gamma$, but in the cases of interest for the present, the decay length is much longer than the detector dimensions.

To estimate the event rate, we consider the up-scattering cross section:

$$
\begin{aligned}
\frac{d \sigma_{\nu e \rightarrow N e}}{d E_{R}}= & d^{2} \alpha Z\left[\frac{1}{E_{R}}-\frac{m_{4}^{2}}{2 E_{\nu} E_{R} m_{e}}\left(1-\frac{E_{R}}{2 E_{\nu}}+\frac{m_{e}}{2 E_{\nu}}\right)\right. \\
& \left.-\frac{1}{E_{\nu}}+\frac{m_{4}^{4}\left(E_{R}-m_{e}\right)}{8 E_{\nu}^{2} E_{R}^{2} m_{e}^{2}}\right]
\end{aligned}
$$

Here, $d$ is the coupling constant defined in Eq. (1), $\alpha$ is the fine structure constant, $m_{e}$ is the electron mass, $m_{4}$ is the mass of the heavy sterile neutrino, $Z$ is the atomic number,
$E_{\nu}$ is the incoming neutrino energy, and $E_{R}$ is the electronrecoil energy.

The electron-recoil spectrum of the up-scattering events can be determined as

$$
\frac{d R^{i}}{d E_{R}}=\mathrm{MT} \times \int_{E_{\nu}^{\min }} \frac{d \Phi_{\nu}^{i}}{d E_{\nu}} \frac{d \sigma_{\nu e \rightarrow N e}^{i}}{d E_{R}}\left(E_{\nu}, E_{R}\right) d E_{\nu},
$$

where $i=\tau$ for the tau neutrino flavor only (unlike the case in [11]). In addition, $\Phi_{\nu}$ is the solar neutrino flux and MT is the exposure. The minimum energy of the incoming neutrino to up-scatter to the $m_{4}$-mass state, yielding electron recoil with an energy $E_{R}$, is

$$
E_{\nu}^{\min }\left(E_{R}\right)=\frac{m_{4}^{2}+2 m_{e} E_{R}}{2\left[\sqrt{E_{R}\left(E_{R}+2 m_{e}\right)}-E_{R}\right]} .
$$

We also include the energy-dependent efficiency given in Fig. 2 of Ref. [1], and include their background estimations in our fits.

\section{RESULTS}

As a first example, we illustrate our best-fit to the spectrum of recoil events at XENON1T in Fig. 1. We display our main results in Fig. 3 assuming only a $\nu_{\tau}$

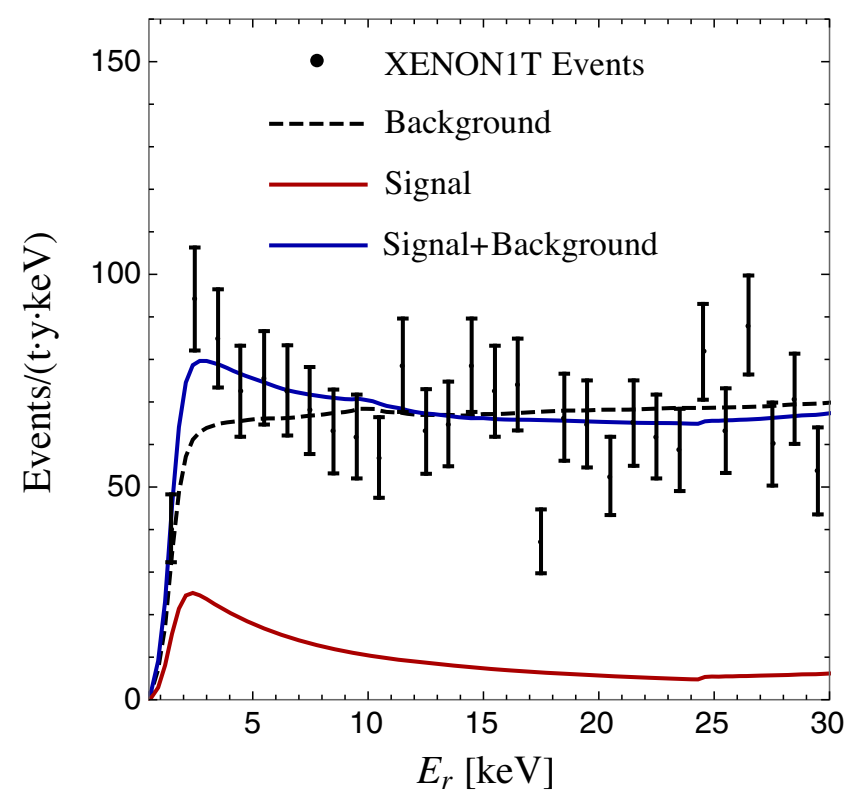

FIG. 1. Dipole portal best fit signal spectrum at XENON1T with $m_{4}=640 \mathrm{keV}$ and $d=2.2 \times 10^{-9} \mu_{B}$. The background is shown in dashed black, the signal is solid red, and the signal plus best-fit background is shown in solid blue. Note that the signal plus best fit background curve dips below the background only curve at higher recoil energies due to the fact that the best fit model includes a background which is suppressed by systematic uncertainties. Included in these event rates are the energydependent signal efficiency. 
coupling for the dipole interaction in Eq. (1). We utilize a $\Delta \chi^{2}$ method to quantify the best-fit parameter significance. The minimum $\chi^{2}$ value is found on the coupling constant $d$ and heavy sterile neutrino mass $m_{4}$ parameter space. $\Delta \chi^{2}$ values for 2 degrees of freedom are used to draw $1 \sigma$ to $7 \sigma$ contours. Note that the $10 \%$ systematic uncertainty from the $\mathrm{Pb}^{214}$ branching ratio is the dominant of the systematic uncertainties in the background event rate [1]. The discovery significance may be lower than we have stated because of the look-elsewhere effect (due to our selective sampling of data in the XENON1T low energy bins only), but we leave a more detailed statistical analysis for further research. Along with the XENON1T contours we display the SN1987A [8] and previous XENON1T bounds based on nuclear recoil data [11]. At larger couplings, the dipole is constrained by LEP [8] and DONUT $[10,80]$.

The equivalent plot for $\mu$-flavor coupling includes strong bounds from CHARM-II at the $3 \times 10^{-9} \mu_{B}$ level. Moreover, Borexino probes up to $m_{4} \simeq 230 \mathrm{keV}$ [10] at the $2.8 \times 10^{-11} \mu_{B}$ level for all flavors [81]. Thus boron- 8 region will survive in the muon-coupling case as well.

\section{EXISTING CONSTRAINTS}

\section{A. Xenon 1T NR constraint}

Previous work utilized XENON1T's nuclear recoil search for DM [82] to place conservative constraints on the neutrino dipole portal interaction [11]. In that case, the low-energy solar neutrinos scatter coherently such that there is a $\sim Z^{2}$ enhancement in the cross section. This analysis [11] specifically vetoed events if the produced heavy sterile decayed inside the detector volume since this would produce an altered ionization/scintillation signal. Despite this reduction in event rate, the bounds derived from the XENON1T nuclear recoil data [82] were stronger than existing bounds on the tau-flavored dipole portal coupling for sterile masses less than $10 \mathrm{MeV}$.

\section{B. Stellar energy loss}

The heavy sterile neutrino $N$ we consider can carry energy when thermally produced in the stellar systems, affecting the energy loss, thermal conductivity, and eventually time evolution of well known stellar populations (see, e.g., [83-85]). When $m_{4}>T_{\text {star }}\left(T_{\text {star }}\right.$ is the temperature of the star) the thermal-averaged energy loss is proportional to $\exp \left(-m_{4} / T_{\text {star }}\right)$ [86]. Taking the powerful production of $N$ in the red-giants into account, our parameter space of interest of a heavy sterile neutrino $N$ below $\sim 250 \mathrm{keV}$ would be constrained, as indicated by the red dashed vertical line in Fig. 3.

We note that if the parameter space is reduced to exclude the low mass region $m_{4}<250 \mathrm{keV}$, the minimum $\chi^{2}$ value is changed due to the exclusion of the best-fit region around $m_{4}=100 \mathrm{keV}$. The significance of the parameter space around $m 4=640 \mathrm{keV}$ (due to the ${ }^{8} \mathrm{~B}$ neutrino flux) is therefore increased.

\section{Supernova 1987A}

The constraints from Supernova 1987A (SN1987A) on a dipole portal heavy sterile neutrino is conducted in [8]. The constraint in our parameter space of interest is enclosed by an upper limit and a lower limit. The lower limit is set by the minimal emission of sterile neutrino $N$ that would carry out enough energy to affect the standard supernovae cooling through neutrinos from the core. The relevant production processes include neutrino upscattering to $N$ with electrons/positrons/protons, $e^{+} e^{-}$annihilating into $N \bar{\nu}$, and neutrino-photon inverse decay. The higher limit of the bound is set by the "trapping" of the sterile neutrino $N$, meaning that the energy carried by $N$ can be recycled and re-emitted within the "neutrinosphere" (an isotherm-sphere within which the neutrino is diffusive rather than free-streaming) [87], and the constraint from supernova cooling can be avoided with large enough coupling. The relevant processes for this trapping consideration are $N$ downscattering to neutrinos with electrons/ positrons/protons, $N-\bar{\nu}$ annihilation to electron pairs, $N$ decay, and gravitational trapping (which kick in at above $\sim 300 \mathrm{MeV}$ ). The constrained regime is shown in Fig. 3.

Notice that the SN1987A bound is subject to large uncertainties in both the supernovae property, the SN1987A measurement, and also the cooling model [88]. In [8], the bound is derived for muon-neutrino dipole; tau-neutrino dipole should be subject to the same bound if one assumes muon and tau neutrinos have the same fluxes (see, e.g., [89]). Improvement of this bound is possible with a better knowledge of the complicated flavor composition and energy spectra of neutrinos during supernovae processes.

\section{Cosmological bounds}

Dark-sector particles of mass around or below a MeV that couple to the SM sector could be subject to strong big bang nucleosynthesis (BBN) constraints [90-92]. Previous work [8] found that BBN constraints on the heavy sterile neutrino with a dipole portal are sensitive to the reheating temperature for a broad range of sterile masses. If $N$ is thermally populated in the early universe, it could have various effects on BBN. In particular, it could contribute to $N_{\text {eff }}$ as a semi-relativistic particle during BBN, and it could decay to photons and neutrinos, affecting their temperatures (also affecting $N_{\text {eff }}$ ) as well as light-element production $[90,91]$.

The major thermalization processes of $N$ are the $2 \rightarrow 2$ processes $e^{+}+e^{-} \rightarrow N+\bar{\nu}$ (synthesis) and $e^{-}+\nu \rightarrow$ $e^{-}+N$ (up-scattering), and neutrino-photon inverse decay, $\nu+\gamma \rightarrow N$. Since the 2 to 2 processes scale as $\Gamma_{2 \rightarrow 2} \propto d^{2} T^{3}$, $N$ will inevitably be thermalized when the SM temperature 


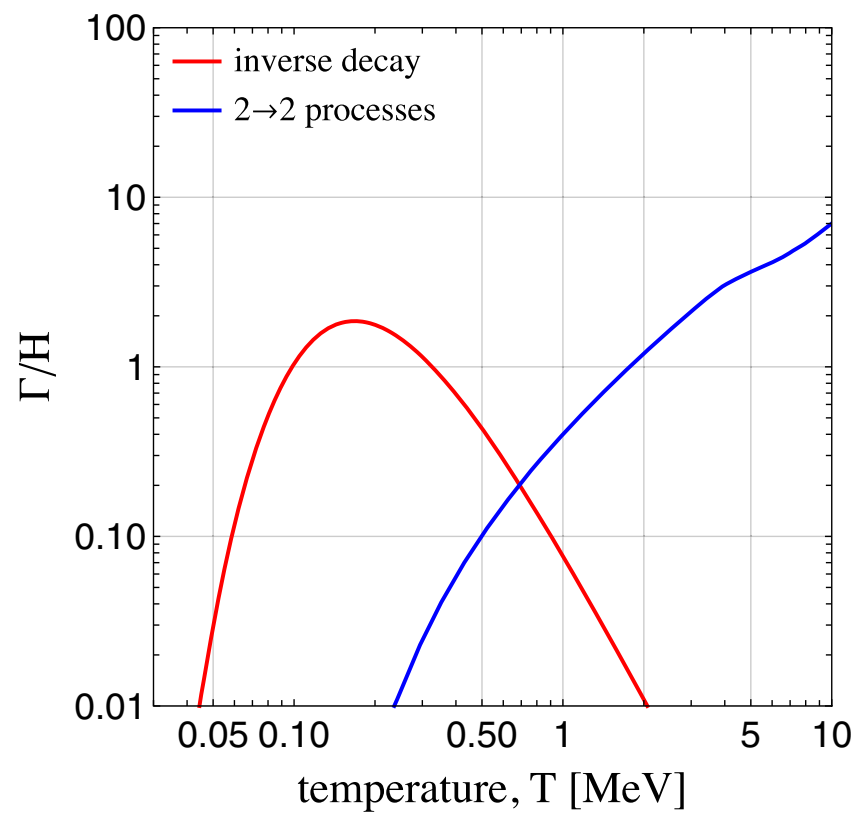

FIG. 2. Comparison of the thermalization rates (inverse decay and $2 \rightarrow 2$ upscattering/synthesis (see text for descriptions), based on the thermal-averaged cross sections and number density, to the temperature-dependent Hubble rate, for the a benchmark parameter point from Fig. $3, m_{4}=600 \mathrm{keV}$ and $d=10^{-9} \mu_{B}$.

$T$ is much higher than the energy scale $d^{-1}$. Under the assumption of high reheating temperature $\left(T_{\mathrm{RH}}\right.$ much larger than $d^{-1}$ ), the sub-MeV region of interest would be strongly constrained.

However, one can consider cosmological scenarios with a very low reheating temperature [93-100], around 2 to $5 \mathrm{MeV}$, and determine if $N$ will be thermalized below such temperatures. In the parameter space that $N$ is not thermalized, the strong BBN bound can be alleviated. In Fig. 2, we show the ratio of the major thermalization processes to the Hubble rate, for a benchmark point in the best fit region for ${ }^{8} \mathrm{~B}$. These rates are calculated through proper thermal-averaging (briefly described in [8]). We also checked that the numerically calculated thermalaveraged cross section and the analytical cross sections taking into account boost factors from the temperature match quantitatively. As one can see, the neutrino-photon inverse decay can play a major role in thermalization with a low-reheating temperature, as it has a resonance region at around $T \sim m_{4} / 2$. In Fig. 2, one can see the behavior of $\Gamma / H$ in different $\mathrm{SM}$ temperatures. Note that $\Gamma / H \sim 1$ is just an estimation of thermalization. Detailed consideration should be conducted to determine the actual thermalization conditions.

In Fig. 3, we display the calculated thermalization curves for the parameter space of interest. Above the upper (lower) dashed gray curves is the parameter space in which the $N$ thermalization rate exceeds the Hubble rate below $2 \mathrm{MeV}(5 \mathrm{MeV})$ temperatures. The $\mathrm{BBN}$

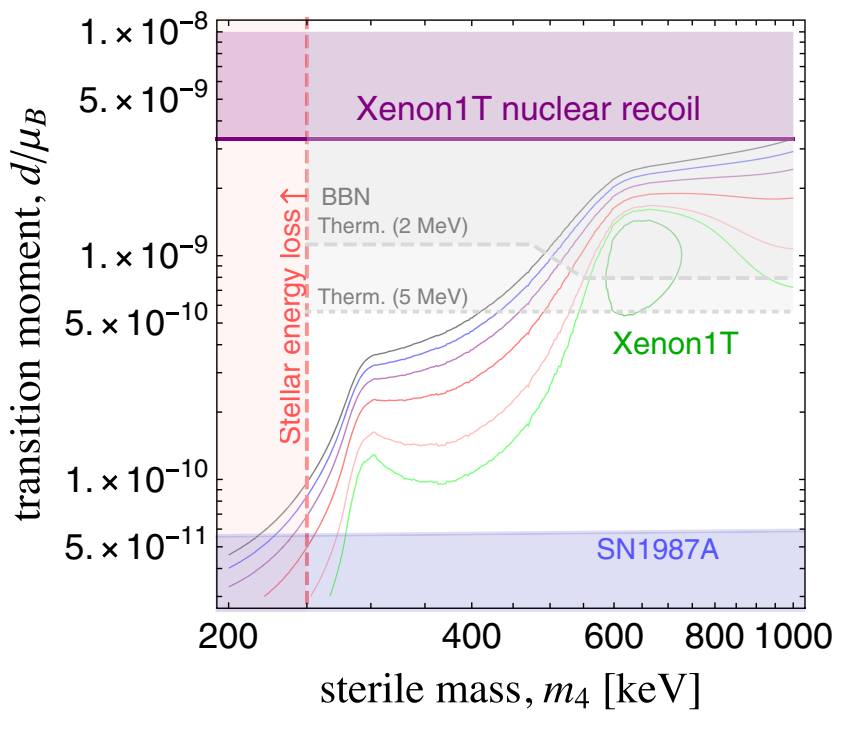

FIG. 3. The XENON1T best-fit contours are drawn from $1 \sigma$ to $7 \sigma$ (propagating outward from the island at $\sim 600 \mathrm{keV}, 10^{-9} \mu_{B}$ ). We also display the SN1987A [8], stellar energy loss, and XENON1T nuclear recoil bounds [11]. We also use dashed/ dotted gray curves to indicate the parameter space in which thermalization (thermal processes exceeding the Hubble rate) could happen at a temperature below $2 \mathrm{MeV}$ (region above the upper dashed-gray) and $5 \mathrm{MeV}$ (region above the lower dottedgray), see texts for detail.

constraints may be alleviated below these curves. Note that the $N$ thermalization processes mentioned above also provide new channels for electron/photon and neutrino sectors to thermalize (even if the $N$ itself is not being fully thermally populated). However, the parameter space below the "thermalization curves" in Fig. 3 still indicate that these interaction rates never exceed the Hubble rate under the low reheating temperatures we have considered ( 2 and $5 \mathrm{MeV}$ ), and that these rates may not be strong enough to directly affect neutrino decoupling or BBN. We find some of the best-fit regions for ${ }^{8} \mathrm{~B}$ flux are covered by $2 \mathrm{MeV}$ or $5 \mathrm{MeV}$ thermalization curves. Given the borderline case for the ${ }^{8} \mathrm{~B}$ region, we believe that a detailed analysis should be conducted to determine the full effects of this portal on neutrino decoupling and BBN. We leave this consideration for a future work.

\section{CONCLUSIONS}

We have shown that the neutrino dipole portal could potentially reconcile a solar neutrino origin with astrophysical and terrestrial bounds with the introduction of a new mass scale sterile neutrino. We also set a new constraint on the model based on the Xenon 1T data. Future probes of the model include: scintillation-only LXe data [101], SHiP [8], DUNE, and a detailed BBN analysis. We further note that direct atmospheric production of sterile neutrinos via the dipole operator may lead to additional 
constraints [102]. Although such models may induce mass mixing with the active neutrinos, these bounds are weak [14-18] for the tau-flavor couplings we focus on.

\section{ACKNOWLEDGMENTS}

We thank Nikita Blinov, Pilar Coloma, Gordan Krnjaic, Ryan Plestid, Maxim Pospelov, Evan Shockley, and Luca Vecchi for useful discussions.
The work of I.M.S. is supported by the U.S. Department of Energy under the Award No. DESC0020250. This document was prepared by Y.-D. T. using the resources of the Fermi National Accelerator Laboratory (Fermilab), a U.S. Department of Energy, Office of Science, HEP User Facility. Fermilab is managed by Fermi Research Alliance, LLC (FRA), acting under Contract No. DE-AC02-07CH11359.
[1] E. Aprile et al. (XENON Collaboration), Phys. Rev. D 102, 072004 (2020).

[2] S. N. Gninenko, Phys. Rev. Lett. 103, 241802 (2009).

[3] S. N. Gninenko, Phys. Rev. D 83, 015015 (2011).

[4] D. McKeen and M. Pospelov, Phys. Rev. D 82, 113018 (2010).

[5] M. Masip and P. Masjuan, Phys. Rev. D 83, 091301 (2011).

[6] S. N. Gninenko, Phys. Lett. B 710, 86 (2012).

[7] M. Masip, P. Masjuan, and D. Meloni, J. High Energy Phys. 01 (2013) 106.

[8] G. Magill, R. Plestid, M. Pospelov, and Y.-D. Tsai, Phys. Rev. D 98, 115015 (2018).

[9] E. Bertuzzo, S. Jana, P. A. Machado, and R. Zukanovich Funchal, Phys. Rev. Lett. 121, 241801 (2018).

[10] P. Coloma, P. A. N. Machado, I. Martinez-Soler, and I. M. Shoemaker, Phys. Rev. Lett. 119, 201804 (2017).

[11] I. M. Shoemaker and J. Wyenberg, Phys. Rev. D 99, 075010 (2019).

[12] K. Fujikawa and R. Shrock, Phys. Rev. Lett. 45, 963 (1980).

[13] R. E. Shrock, Nucl. Phys. B206, 359 (1982).

[14] J. C. Helo, S. Kovalenko, and I. Schmidt, Phys. Rev. D 84, 053008 (2011).

[15] D. Bryman and R. Shrock, Phys. Rev. D 100, 073011 (2019).

[16] C. Dib, J. Helo, M. Nayak, N. Neill, A. Soffer, and J. Zamora-Saa, Phys. Rev. D 101, 093003 (2020).

[17] C. Kim, D. H. Lee, S. Oh, and D. Sahoo, Eur. Phys. J. C 80, 730 (2020).

[18] D. Bryman and R. Shrock, Phys. Rev. D 100, 053006 (2019).

[19] M. Agostini et al. (Borexino Collaboration), Eur. Phys. J. C 80, 1091 (2020).

[20] F. Takahashi, M. Yamada, and W. Yin, Phys. Rev. Lett. 125, 161801 (2020).

[21] K. Kannike, M. Raidal, H. Veermäe, A. Strumia, and D. Teresi, Phys. Rev. D 102, 095002 (2020).

[22] G. Alonso-Álvarez, F. Ertas, J. Jaeckel, F. Kahlhoefer, and L. Thormaehlen, J. Cosmol. Astropart. Phys. 11 (2020) 029.

[23] D. W. P. Amaral, D. G. Cerdeno, P. Foldenauer, and E. Reid, J. High Energy Phys. 12 (2020) 155.

[24] B. Fornal, P. Sandick, J. Shu, M. Su, and Y. Zhao, Phys. Rev. Lett. 125, 161804 (2020).

[25] C. Boehm, D. G. Cerdeno, M. Fairbairn, P. A. Machado, and A. C. Vincent, Phys. Rev. D 102, 115013 (2020).
[26] K. Harigaya, Y. Nakai, and M. Suzuki, Phys. Lett. B 809, 135729 (2020).

[27] A. Bally, S. Jana, and A. Trautner, arXiv:2006.11919.

[28] L. Su, W. Wang, L. Wu, J. M. Yang, and B. Zhu, Phys. Rev. D 102, 115028 (2020).

[29] M. Du, J. Liang, Z. Liu, V. Q. Tran, and Y. Xue, Chin. Phys. C 45, 013114 (2021).

[30] L. Di Luzio, M. Fedele, M. Giannotti, F. Mescia, and E. Nardi, Phys. Rev. Lett. 125, 131804 (2020).

[31] N. F. Bell, J. B. Dent, B. Dutta, S. Ghosh, J. Kumar, and J. L. Newstead, Phys. Rev. Lett. 125, 161803 (2020).

[32] Y. Chen, J. Shu, X. Xue, G. Yuan, and Q. Yuan, J. High Energy Phys. 04 (2021) 282.

[33] U. K. Dey, T. N. Maity, and T. S. Ray, Phys. Lett. B 811, 135900 (2020).

[34] G. Choi, M. Suzuki, and T. T. Yanagida, Phys. Lett. B 811, 135976 (2020).

[35] D. Aristizabal Sierra, V. De Romeri, L. Flores, and D. Papoulias, Phys. Lett. B 809, 135681 (2020).

[36] J. Buch, M. A. Buen-Abad, J. Fan, and J. S. C. Leung, J. Cosmol. Astropart. Phys. 10 (2020) 051.

[37] G. Paz, A. A. Petrov, M. Tammaro, and J. Zupan, Phys. Rev. D 103, L051703 (2021).

[38] H. M. Lee, J. High Energy Phys. 01 (2021) 019.

[39] Q.-H. Cao, R. Ding, and Q.-F. Xiang, Chin. Phys. C 45, 045002 (2021).

[40] A. E. Robinson, arXiv:2006.13278.

[41] A. N. Khan, Phys. Lett. B 809, 135782 (2020).

[42] R. Primulando, J. Julio, and P. Uttayarat, Eur. Phys. J. C 80, 1084 (2020).

[43] K. Nakayama and Y. Tang, Phys. Lett. B 811, 135977 (2020).

[44] Y. Jho, J.-C. Park, S. C. Park, and P.-Y. Tseng, Phys. Lett. B 811, 135863 (2020).

[45] J. Bramante and N. Song, Phys. Rev. Lett. 125, 161805 (2020).

[46] M. Baryakhtar, A. Berlin, H. Liu, and N. Weiner, arXiv: 2006.13918.

[47] H. An, M. Pospelov, J. Pradler, and A. Ritz, Phys. Rev. D 102, 115022 (2020).

[48] L. Zu, G.-W. Yuan, L. Feng, and Y.-Z. Fan, Nucl. Phys. B965, 115369 (2021).

[49] C. Gao, J. Liu, L.-T. Wang, X.-P. Wang, W. Xue, and Y.-M. Zhong, Phys. Rev. Lett. 125, 131806 (2020). 
[50] R. Budnik, H. Kim, O. Matsedonskyi, G. Perez, and Y. Soreq, Phys. Rev. D 104, 015012 (2021).

[51] M. Lindner, Y. Mambrini, T. B. de Melo, and F. S. Queiroz, Phys. Lett. B 811, 135972 (2020).

[52] I. M. Bloch, A. Caputo, R. Essig, D. Redigolo, M. Sholapurkar, and T. Volansky, J. High Energy Phys. 01 (2021) 178.

[53] W. DeRocco, P. W. Graham, and S. Rajendran, Phys. Rev. D 102, 075015 (2020).

[54] J. B. Dent, B. Dutta, J. L. Newstead, and A. Thompson, Phys. Rev. Lett. 125, 131805 (2020).

[55] K. Zioutas, G. Cantatore, M. Karuza, A. Kryemadhi, M. Maroudas, and Y. Semertzidis, arXiv:2006.16907.

[56] D. McKeen, M. Pospelov, and N. Raj, Phys. Rev. Lett. 125, 231803 (2020).

[57] P. Coloma, P. Huber, and J. M. Link, arXiv:2006.15767.

[58] H. An and D. Yang, Phys. Lett. B 818, 136408 (2021).

[59] L. Delle Rose, G. Hütsi, C. Marzo, and L. Marzola, J. Cosmol. Astropart. Phys. 02 (2021) 031.

[60] S.-F. Ge, P. Pasquini, and J. Sheng, Phys. Lett. B 810, 135787 (2020).

[61] B. Bhattacherjee and R. Sengupta, Phys. Lett. B 817, 136305 (2021).

[62] C. Dessert, J. W. Foster, Y. Kahn, and B. R. Safdi, Phys. Dark Universe 34, 100878 (2021).

[63] W. Chao, Y. Gao, and M.j. Jin, arXiv:2006.16145.

[64] G. Cacciapaglia, C. Cai, M. T. Frandsen, M. Rosenlyst, and H. H. Zhang, Phys. Rev. D 102, 075018 (2020).

[65] P. Ko and Y. Tang, Phys. Lett. B 815, 136181 (2021).

[66] Y. Gao and T. Li, arXiv:2006.16192.

[67] H. Alhazmi, D. Kim, K. Kong, G. Mohlabeng, J.-C. Park, and S. Shin, J. High Energy Phys. 05 (2021) 055.

[68] S. Baek, J. Kim, and P. Ko, Phys. Lett. B 810, 135848 (2020).

[69] T. Li, arXiv:2007.00874.

[70] S. Chigusa, M. Endo, and K. Kohri, J. Cosmol. Astropart. Phys. 10 (2020) 035.

[71] O. Miranda, D. Papoulias, M. Tórtola, and J. Valle, Phys. Lett. B 808, 135685 (2020).

[72] K. Benakli, C. Branchina, and G. Lafforgue-Marmet, Eur. Phys. J. C 80, 1118 (2020).

[73] N. Okada, S. Okada, D. Raut, and Q. Shafi, Phys. Lett. B 810, 135785 (2020).

[74] G. Choi, T. T. Yanagida, and N. Yokozaki, Phys. Lett. B 810, 135836 (2020).

[75] J. Smirnov and J. F. Beacom, Phys. Rev. Lett. 125, 131301 (2020).

[76] M. Chala and A. Titov, J. High Energy Phys. 09 (2020) 188.

[77] K. Babu, S. Jana, and M. Lindner, J. High Energy Phys. 10 (2020) 040.
[78] D. Croon, S. D. McDermott, and J. Sakstein, Phys. Dark Universe 32, 100801 (2021).

[79] A. Bally, S. Jana, and A. Trautner, Phys. Rev. Lett. 125, 161802 (2020).

[80] R. Schwienhorst et al. (DONUT Collaboration), Phys. Lett. B 513, 23 (2001).

[81] M. Agostini et al. (Borexino Collaboration), Phys. Rev. D 96, 091103 (2017).

[82] E. Aprile et al. (XENON Collaboration), Phys. Rev. Lett. 121, 111302 (2018).

[83] G. Raffelt and A. Weiss, Phys. Rev. D 51, 1495 (1995).

[84] S. Arceo-Díaz, K.-P. Schröder, K. Zuber, and D. Jack, Astropart. Phys. 70, 1 (2015).

[85] S. A. Díaz, K.-P. Schröder, K. Zuber, D. Jack, and E. E. B. Barrios, arXiv:1910.10568.

[86] M. Pospelov and Y.-D. Tsai, Phys. Lett. B 785, 288 (2018).

[87] J. H. Chang, R. Essig, and S. D. McDermott, J. High Energy Phys. 01 (2017) 107.

[88] N. Bar, K. Blum, and G. D'amico, Phys. Rev. D 101, 123025 (2020).

[89] T. Fischer, S. C. Whitehouse, A. Mezzacappa, F.-K. Thielemann, and M. Liebendörfer, Astron. Astrophys. 517, A80 (2010).

[90] Z. Berezhiani, A. Dolgov, and I. Tkachev, J. Cosmol. Astropart. Phys. 02 (2013) 010.

[91] C. Boehm, M. J. Dolan, and C. McCabe, J. Cosmol. Astropart. Phys. 08 (2013) 041.

[92] G. Krnjaic and S. D. McDermott, Phys. Rev. D 101, 123022 (2020).

[93] T. Moroi and L. Randall, Nucl. Phys. B570, 455 (2000).

[94] S. Hannestad, Phys. Rev. D 70, 043506 (2004).

[95] K. Kohri, T. Moroi, and A. Yotsuyanagi, Phys. Rev. D 73, 123511 (2006).

[96] J. Pradler and F. D. Steffen, Phys. Lett. B 648, 224 (2007).

[97] P. de Salas, M. Lattanzi, G. Mangano, G. Miele, S. Pastor, and O. Pisanti, Phys. Rev. D 92, 123534 (2015).

[98] A. Berlin, N. Blinov, G. Krnjaic, P. Schuster, and N. Toro, Phys. Rev. D 99, 075001 (2019).

[99] T. Hasegawa, N. Hiroshima, K. Kohri, R. S. Hansen, T. Tram, and S. Hannestad, J. Cosmol. Astropart. Phys. 12 (2019) 012.

[100] T. Hasegawa, N. Hiroshima, K. Kohri, R. S. Hansen, T. Tram, and S. Hannestad, J. Cosmol. Astropart. Phys. 08 (2020) 015.

[101] E. Aprile et al. (XENON Collaboration), Phys. Rev. Lett. 123, 251801 (2019).

[102] A. Bueno, M. Masip, P. Sánchez-Lucas, and N. Setzer, Phys. Rev. D 88, 073010 (2013). 